\title{
Drift estimation for a Lévy-driven Ornstein-Uhlenbeck process with heavy tails
}

\author{
Alexander Gushchin ${ }^{1,2} \cdot$ Ilya Pavlyukevich ${ }^{3} \cdot$ Marian Ritsch $^{3}$
}

Received: 25 November 2019 / Accepted: 18 March 2020 / Published online: 27 March 2020

(c) The Author(s) 2020

\section{Abstract}

We consider the problem of estimation of the drift parameter of an ergodic OrnsteinUhlenbeck type process driven by a Lévy process with heavy tails. The process is observed continuously on a long time interval $[0, T], T \rightarrow \infty$. We prove that the statistical model is locally asymptotic mixed normal and the maximum likelihood estimator is asymptotically efficient.

Keywords Lévy process · Ornstein-Uhlenbeck type process · Local asymptotic mixed normality $\cdot$ Heavy tails $\cdot$ Regular variation · Maximum likelihood estimator $\cdot$ Asymptotic observed information

Mathematics Subject Classification 62M05 $\cdot 60 \mathrm{~F} 05 \cdot 60 \mathrm{~J} 75$

\section{Introduction, motivation, previous results}

In this paper, we deal with an estimation of the drift parameter $\theta>0$ of an ergodic onedimensional Ornstein-Uhlenbeck process $X$ driven by a Lévy process:

$$
X_{t}=X_{0}-\theta \int_{0}^{t} X_{s} \mathrm{~d} s+Z_{t}, \quad t \geq 0 .
$$

The process $Z$ is a one-dimensional Lévy process with known characteristics and with infinite variance. The process $X$ is observed continuously on a long time interval $[0, T], T \rightarrow \infty$.

\footnotetext{
$\triangle \quad$ Ilya Pavlyukevich

ilya.pavlyukevich@uni-jena.de

Alexander Gushchin

gushchin@mi-ras.ru

Marian Ritsch

carl.christian.marian.ritsch@uni-jena.de

1 Steklov Mathematical Institute of Russian Academy of Sciences, 8 Gubkina St., Moscow, Russia 119991

2 National Research University Higher School of Economics, Moscow, Russia

3 Institute of Mathematics, Friedrich Schiller University Jena, Ernst-Abbe-Platz 2, 07743 Jena, Germany
} 
The problem is to study asymptotic properties of the corresponding statistical model and to show that the maximum likelihood estimator of $\theta$ is asymptotically efficient in an appropriate sense. Although the continuous time observations are far from being realistic in applications, they are of theoretical importance since they can be considered as a limit of high frequency discrete models.

Since we deal with continuous observations, it is natural to assume that the Gaussian component of the Lévy process $Z$ is not degenerate. In this case, the laws of observations corresponding to different values of $\theta$ are equivalent and the likelihood ratio has an explicit form.

There are a lot of papers devoted to inference for Lévy driven SDEs. Most of the literature treats the case of discrete time observations both in the high and low frequency setting. A general theory for the likelihood inference for continuously observed jump-diffusions can be found in Sørensen (1991).

A complete analysis of the drift estimation for continuously observed ergodic and nonergodic Ornstein-Uhlenbeck process driven by a Brownian motion can be found in Höpfner (2014, Chapter 8.1).

For continuously observed square integrable Lévy driven Ornstein-Uhlenbeck processes, the local asymptotic normality (LAN) of the model and the asymptotic efficiency of the maximum likelihood estimator of the drift have been derived by Mai $(2012,2014)$ with the help of the theory of exponential families, see Küchler and Sørensen (1997).

High frequency estimation of a square integrable Lévy driven Ornstein-Uhlenbeck process with non-vanishing Gaussian component has been performed by Mai (2012, 2014). Kawai (2013) studied the asymptotics of the Fisher information for three characterizing parameters of Ornstein-Uhlenbeck processes with jumps under low frequency and high frequency discrete sampling. The existence of all moments of the Lévy process was assumed. Tran (2017) considered the ergodic Ornstein-Uhlenbeck process driven by a Brownian motion and a compensated Poisson process, whose drift and diffusion coefficients as well as its jump intensity depend on unknown parameters. He obtained the LAN property of the model in the high frequency setting.

We also mention the works by Hu and Long (2007, 2009a, b), Long (2009) and Zhang and Zhang (2013) devoted to the least-square estimation of parameters of the Ornstein-Uhlenbeck process driven by an $\alpha$-stable Lévy process.

There is vast literature devoted to parametric inference for discretely observed Lévy processes (see, e.g. a survey by Masuda (2015)) and Lévy driven SDEs. More results on the latter topic can be found e.g. in Masuda (2013), Ivanenko and Kulik (2014), Kohatsu-Higa et al. (2017), Masuda (2019), Uehara (2019), Clément and Gloter (2015), Clément et al. (2019), Clément and Gloter (2019), Nguyen (2018) and Gloter et al. (2018) and the references therein.

In this paper, we fill the gap and analyse a continuously observed ergodic OrnsteinUhlenbeck process driven by a Lévy process with heavy regularly varying tails of index $-\alpha$, $\alpha \in(0,2)$, in the presence of a Gaussian component. It turns out that the log-likelihood in this model is quadratic, however the model is not asymptotically normal and we prove only the local asymptotic mixed normality (LAMN) property. We refer to Le Cam and Yang (2000) and Höpfner (2014) for the general theory of estimation for LAMN models.

The fact that the prelimiting log-likelihood is quadratic automatically implies that the maximum likelihood estimator is asymptotically efficient in the sense of Jeganathan's convolution theorem and attains the local asymptotic minimax bound. Another feature of our model is that the asymptotic observed information has spectrally positive $\alpha / 2$-stable distribution. This implies that the limiting law of the maximum likelihood estimator has tails of the order $\exp \left(-x^{\alpha}\right)$ and hence finite moments of all orders. 
The paper is organized as follows. In the next section we formulate the assumptions of our model and the main results of the paper. Section 3 contains auxiliary results that will be used in the proof of the main Theorem 2.5. In particular, we calculate the tail of a product of two iid heavy-tail random variables (Lemma 3.2), a conditional law of inter-arrival times of a Poisson process, and prove a technically involved Lemma 3.7. Eventually in Sect. 4, the proofs of the main results are presented.

\section{Setting and the main result}

Consider a stochastic basis $(\Omega, \mathscr{F}, \mathbb{F}, \mathbf{P}), \mathbb{F}$ being right-continuous. Let $Z$ be a Lévy process with the characteristic triplet $\left(\sigma^{2}, b, v\right)$ and the Lévy-Itô decomposition

$$
Z_{t}=\sigma W_{t}+b t+\int_{0}^{t} \int_{|z| \leq 1} z \tilde{N}(\mathrm{~d} z, \mathrm{~d} s)+\int_{0}^{t} \int_{|z|>1} z N(\mathrm{~d} z, \mathrm{~d} s),
$$

where $W$ is a standard one-dimensional Brownian motion, $N$ is a Poisson random measure on $\mathbb{R} \backslash\{0\}$ with the Lévy measure $v$ satisfying $\int_{\mathbb{R}}\left(z^{2} \wedge 1\right) v(\mathrm{~d} z)<\infty, \tilde{N}$ is the compensated Poisson random measure, and $b \in \mathbb{R}$.

For $\theta \in \mathbb{R}$, let $X$ be an Ornstein-Uhlenbeck type process being a solution of the SDE

$$
X_{t}=X_{0}-\theta \int_{0}^{t} X_{s} \mathrm{~d} s+Z_{t}, \quad t \geq 0,
$$

where $\theta \in \mathbb{R}$ is an unknown parameter. The initial value $X_{0} \in \mathscr{F}_{0}$ is a random variable whose distribution does not depend on $\theta$. Note that $X$ has an explicit representation

$$
X_{t}=X_{0} \mathrm{e}^{-\theta t}+\int_{0}^{t} \mathrm{e}^{-\theta(t-s)} \mathrm{d} Z_{s}, \quad t \geq 0,
$$

see, e.g. Applebaum (2009, Sections 4.3.5 and 6.3) and Sato (1999, Section 17).

Let $\mathbb{D}=D([0, \infty), \mathbb{R})$ be the space of real-valued càdlàg functions $\omega:[0, \infty) \rightarrow \mathbb{R}$ equipped with Skorokhod topology and Borel $\sigma$-algebra $\mathscr{B}(\mathbb{D})$. The space $(\mathbb{D}, \mathscr{B}(\mathbb{R}))$ is Polish, and $\mathscr{B}(\mathbb{D})$ coincides with the $\sigma$-algebra generated by the coordinate projections. We define a (right-continuous) filtration $\mathbb{G}=\left(\mathscr{G}_{t}\right)_{t \geq 0}$ consisting of $\sigma$-algebras

$$
\mathscr{G}_{t}:=\bigcap_{s>t} \sigma\left(\omega_{r}: r \leq s, \omega \in \mathbb{D}\right), \quad t \geq 0 .
$$

For each $\theta \in \mathbb{R}$, the process $X=\left(X_{t}\right)_{t \geq 0}$ induces a measure $\mathbf{P}^{\theta}$ on the path space $(\mathbb{D}, \mathscr{B}(\mathbb{D}))$. Let

$$
\mathbf{P}_{T}^{\theta}=\left.\mathbf{P}^{\theta}\right|_{\mathscr{G}_{T}}
$$

be a restriction of $\mathbf{P}^{\theta}$ to the $\sigma$-algebra $\mathscr{G}_{T}$.

In order to establish the equivalence of the laws $\mathbf{P}_{T}^{\theta}$ and $\mathbf{P}_{T}^{\theta_{0}}, \theta, \theta_{0} \in \mathbb{R}$, we have to make the following assumption.

$\mathbf{A}_{\sigma}$ : The Brownian component of $Z$ is non-degenerate, i.e. $\sigma>0$.

Proposition 2.1 Let $\boldsymbol{A}_{\sigma}$ hold true. Then for each $T>0$, any $\theta, \theta_{0} \in \mathbb{R}$

$$
\mathbf{P}_{T}^{\theta} \sim \mathbf{P}_{T}^{\theta_{0}},
$$


and the likelihood ratio is given by

$$
L_{T}\left(\theta_{0}, \theta\right)=\frac{\mathrm{d} \mathbf{P}_{T}^{\theta}}{\mathrm{d} \mathbf{P}_{T}^{\theta_{0}}}=\exp \left(-\frac{\theta-\theta_{0}}{\sigma^{2}} \int_{0}^{T} \omega_{s} \mathrm{~d} m_{s}^{\left(\theta_{0}\right)}-\frac{\left(\theta-\theta_{0}\right)^{2}}{2 \sigma^{2}} \int_{0}^{T} \omega_{s}^{2} \mathrm{~d} s\right),
$$

where

$$
\begin{aligned}
m_{t}^{\left(\theta_{0}\right)}=\omega_{t} & -\omega_{0}+\theta_{0} \int_{0}^{t} \omega_{s} \mathrm{~d} s-b t-\sum_{s \leq t} \Delta \omega_{s} \mathbb{I}\left(\left|\Delta \omega_{s}\right|>1\right) \\
& -\int_{0}^{t} \int_{|x| \leq 1} x(\mu(\mathrm{d} x, \mathrm{~d} s)-v(\mathrm{~d} x) \mathrm{d} s)
\end{aligned}
$$

is the continuous local martingale component of $\omega$ under the measure $\mathbf{P}_{T}^{\theta_{0}}$, and the random measure

$$
\mu(\mathrm{d} x, \mathrm{~d} s)=\sum_{s} \mathbb{I}\left(\Delta \omega_{s} \neq 0\right) \delta_{\left(\Delta \omega_{s}, s\right)}(\mathrm{d} x, \mathrm{~d} s)
$$

is defined by the jumps of $\omega$.

Proof See Jacod and Shiryaev (2003, Theorem III-5-34).

Consider a family of statistical experiments

$$
\left(\mathbb{D}, \mathscr{G}_{T},\left\{\mathbf{P}_{T}^{\theta}\right\}_{\theta>0}\right)_{T>0} .
$$

Our goal is to establish local asymptotic mixed normality (LAMN) of these experiments under the assumption that the process $Z$ has heavy tails. We make the following assumption. $\mathbf{A}_{v}$ : The Lévy measure $v$ has a regularly varying heavy tail of the order $\alpha \in(0,2)$, i.e.

$$
H(R):=\int_{|z|>R} v(\mathrm{~d} z) \in \mathrm{RV}_{-\alpha}, \quad R>0 .
$$

In other words, $H:(0, \infty) \rightarrow(0, \infty)$ and there is a positive function $l=l(R)$ slowly varying at infinity such that

$$
H(R)=\frac{l(R)}{R^{\alpha}}, \quad R>0 .
$$

Let us consider the function

$$
\tilde{H}(R)=\alpha \int_{R}^{\infty} \frac{H(z)}{z} \mathrm{~d} z, \quad R>0 .
$$

Since $H(z)>0, z>0$, the function $\tilde{H}$ is absolutely continuous and strictly decreasing. Moreover, by Karamata's theorem, see e.g. Resnick (2007, Theorem 2.1 (a)), applied to the function $\frac{H(z)}{z} \in \mathrm{RV}_{-\alpha-1}$ we obtain the equivalence

$$
\lim _{R \rightarrow \infty} \frac{\tilde{H}(R)}{H(R)}=\lim _{R \rightarrow \infty} \frac{\alpha \int_{R}^{\infty} \frac{H(z)}{z} \mathrm{~d} z}{H(R)}=1 .
$$

We use the function $\tilde{H}$ to introduce the continuous and monotone increasing scaling $\left\{\phi_{T}\right\}_{T>0}$ defined by the relation

$$
\frac{1}{\phi_{T}}:=\tilde{H}^{-1}\left(\frac{1}{T}\right),
$$

where $\tilde{H}^{-1}(R):=\inf \{u>0: \tilde{H}(u)=R\}$ is the (continuous) inverse of $\tilde{H}$. It is easy to see that $\phi_{T} \in \mathrm{RV}_{-1 / \alpha}$. 
Remark 2.2 We make use of the absolutely continuous and strictly decreasing function $\tilde{H}$ just for convenience in order to avoid technicalities connected with the inversion of càdlàg functions. For instance, one can equivalently define $\phi_{T}:=\left(H^{\leftarrow}(1 / T)\right)^{-1}$ for the generalized inverse $H^{\leftarrow}(R):=\inf \{u>0: H(u)>R\}$, see Bingham et al. (1987, Chapter 1.5.7).

Remark 2.3 By Theorem 17.5 in Sato (1999), for each $\theta>0$ the Ornstein-Uhlenbeck process $X$ has an invariant distribution if $\int_{|z|>1} \ln |z| v(\mathrm{~d} z)<\infty$. The latter inequality easily follows from Assumption $\mathbf{A}_{v}$ and Potter's bounds (3.2).

Example 2.4 Let the jump part of the process $Z$ be an $\alpha$-stable Lévy process, i.e. for $\alpha \in(0,2)$ and $c_{-}, c_{+} \geq 0, c_{-}+c_{+}>0$, let

$$
v(\mathrm{~d} z)=\left(\frac{c_{-}}{|z|^{1+\alpha}} \mathbb{I}(z<0)+\frac{c_{+}}{z^{1+\alpha}} \mathbb{I}(z>0)\right) \mathrm{d} z .
$$

Then

$$
\begin{aligned}
H(R) & =\tilde{H}(R)=\frac{c_{-}+c_{+}}{\alpha R^{\alpha}}, \\
\tilde{H}^{-1}(T) & =\left(\frac{c_{-}+c_{+}}{\alpha}\right)^{1 / \alpha} \frac{1}{T^{1 / \alpha}},
\end{aligned}
$$

and

$$
\phi_{T}=\left(\frac{\alpha}{c_{-}+c_{+}}\right)^{1 / \alpha} \frac{1}{T^{1 / \alpha}} .
$$

The main result is the LAMN property of our model.

Theorem 2.5 Let $\boldsymbol{A}_{\sigma}$ and $\boldsymbol{A}_{v}$ hold true. Then the family of statistical experiments (2.4) is locally asymptotically mixed normal at each $\theta_{0}>0$, namely for each $u \in \mathbb{R}$

$$
\operatorname{Law}\left(\ln L_{T}\left(\theta_{0}, \theta_{0}+\phi_{T} u\right) \mid \mathbf{P}_{T}^{\theta_{0}}\right) \rightarrow \mathcal{N} \sqrt{\frac{\mathcal{S}^{(\alpha / 2)}}{2 \sigma^{2} \theta_{0}}} u-\frac{1}{2} \frac{\mathcal{S}^{(\alpha / 2)}}{2 \sigma^{2} \theta_{0}} u^{2}, \quad T \rightarrow \infty,
$$

where $\mathcal{N}$ is a standard Gaussian random variable and $\mathcal{S}^{(\alpha / 2)}$ is an independent spectrally positive $\alpha / 2$-stable random variable with the Laplace transform

$$
\mathbf{E e}^{-\lambda \mathcal{S}^{(\alpha / 2)}}=\mathrm{e}^{-\Gamma\left(1-\frac{\alpha}{2}\right) \lambda^{\alpha / 2}}, \quad \lambda \geq 0 .
$$

Theorem 2.5 is based on the following key result.

Theorem 2.6 Let $\boldsymbol{A}_{\sigma}$ and $\boldsymbol{A}_{v}$ hold true. Then for each $\theta_{0}>0$

$$
\operatorname{Law}\left(\phi_{T}^{2} \int_{0}^{T} X_{s}^{2} \mathrm{~d} s \mid \mathbf{P}_{T}^{\theta_{0}}\right) \rightarrow \frac{\mathcal{S}^{(\alpha / 2)}}{2 \theta_{0}}, \quad T \rightarrow \infty,
$$

where $\mathcal{S}^{(\alpha / 2)}$ is a random variable with the Laplace transform (2.6).

Corollary 2.7 Let $\boldsymbol{A}_{\sigma}$ and $\boldsymbol{A}_{v}$ hold true. Then for each $\theta_{0}>0$

$$
\operatorname{Law}\left(\phi_{T} \int_{0}^{T} X_{s} \mathrm{~d} W_{s}, \phi_{T}^{2} \int_{0}^{T} X_{s}^{2} \mathrm{~d} s \mid \mathbf{P}_{T}^{\theta_{0}}\right) \rightarrow\left(\mathcal{N} \sqrt{\frac{\mathcal{S}^{(\alpha / 2)}}{2 \theta_{0}}}, \frac{\mathcal{S}^{(\alpha / 2)}}{2 \theta_{0}}\right), \quad T \rightarrow \infty .
$$

Proposition 2.1 and Theorem 2.5 allow us to establish asymptotic distribution of the maximum likelihood estimator $\hat{\theta}_{T}$ of $\theta$. Moreover, the special form of the likelihood ratio guarantees that $\hat{\theta}_{T}$ is asymptotically efficient. 
Corollary 2.8 1. Let $\boldsymbol{A}_{\sigma}$ hold true. Then the maximum likelihood estimator $\hat{\theta}_{T}$ of $\theta$ satisfies

$$
\hat{\theta}_{T}=\theta_{0}-\frac{\int_{0}^{T} \omega_{s} \mathrm{~d} m_{s}^{\left(\theta_{0}\right)}}{\int_{0}^{T} \omega_{s}^{2} \mathrm{~d} s} .
$$

2. Let $\boldsymbol{A}_{\sigma}$ and $\boldsymbol{A}_{v}$ hold true. Then

$$
\operatorname{Law}\left(\frac{\hat{\theta}_{T}-\theta_{0}}{\phi_{T}} \mid \mathbf{P}_{T}^{\theta_{0}}\right) \rightarrow \sigma \sqrt{2 \theta_{0}} \cdot \frac{\mathcal{N}}{\sqrt{\mathcal{S}^{(\alpha / 2)}}}, \quad T \rightarrow \infty
$$

The maximum likelihood estimator $\hat{\theta}_{T}$ is asymptotically efficient in the sense of the convolution theorem and the local asymptotic minimax theorem for LAMN models, see Höpfner (2014, Theorems 7.10 and 7.12).

Remark 2.9 It is instructive to determine the tails of the random variable $\mathcal{N} / \sqrt{\mathcal{S}^{(\alpha / 2)}}$ : for each $\alpha \in(0,2)$

$$
\limsup _{x \rightarrow+\infty} x^{-\alpha} \ln \mathbf{P}\left(\frac{|\mathcal{N}|}{\sqrt{\mathcal{S}^{(\alpha / 2)}}}>x\right)<0
$$

and in particular all moments of the r.h.s. of (2.9) are finite.

\section{Auxiliary results}

We decompose the Lévy process $Z$ into a compound Poisson process with heavy jumps, and the rest. Consider the non-decreasing function $R_{T}=T^{\rho}:[1, \infty) \rightarrow[1, \infty)$, where $\rho \geq 0$ will be chosen later.

Denote

$$
\begin{aligned}
& \eta_{t}^{T}=\int_{0}^{t} \int_{|z|>R_{T}} z N(\mathrm{~d} z, \mathrm{~d} s), \\
& \xi_{t}^{T}=\sigma W_{t}+\int_{0}^{t} \int_{|z| \leq R_{T}} z \tilde{N}(\mathrm{~d} z, \mathrm{~d} s), \\
& b_{T}=b+\int_{1<|z| \leq R_{T}} z v(\mathrm{~d} z), \\
& Z_{t}^{T}=Z_{t}-\eta_{t}^{T}=\xi_{t}^{T}+b_{T} t .
\end{aligned}
$$

For each $T \geq 1$, the process $\eta^{T}$ is a compound Poisson process with intensity $H\left(R_{T}\right)$, the iid jumps $\left\{J_{k}^{T}\right\}_{k \geq 1}$ occurring at arrival times $\left\{\tau_{k}^{T}\right\}_{k \geq 1}$, such that

$$
\begin{aligned}
& \mathbf{P}\left(\left|J_{k}^{T}\right| \geq z\right)=\frac{H(z)}{H\left(R_{T}\right)}, \quad z \geq R_{T}, \\
& \mathbf{P}\left(\tau_{k+1}^{T}-\tau_{k}^{T}>u\right)=\mathrm{e}^{-H\left(R_{T}\right) u}, \quad u \geq 0 .
\end{aligned}
$$

Denote also by $N^{T}$ the Poisson counting process of $\eta^{T}$; it is a Poisson process with intensity $H\left(R_{T}\right)$. 
We decompose the Ornstein-Uhlenbeck process $X$ into a sum

$$
\begin{aligned}
X_{t} & =X_{t}^{T}+X_{t}^{\eta^{T}}, \\
X_{t}^{T}: & =X_{0} \mathrm{e}^{-\theta t}+\int_{0}^{t} \mathrm{e}^{-\theta(t-s)} \mathrm{d} Z_{s}^{T}, \\
X_{t}^{\eta^{T}}: & =\int_{0}^{t} \mathrm{e}^{-\theta(t-s)} \mathrm{d} \eta_{s}^{T} .
\end{aligned}
$$

Since $H(\cdot) \in \mathrm{RV}_{-\alpha}$ and $\phi . \in \mathrm{RV}_{-1 / \alpha}, \alpha \in(0,2)$, by Potter's bounds (see, e.g. Resnick (2007, Proposition 2.6 (ii)) for each $\varepsilon>0$ there are constants $0<c_{\varepsilon} \leq C_{\varepsilon}<\infty$ such that for $u \geq 1$

$$
\begin{aligned}
& \frac{c_{\varepsilon}}{u^{\alpha+\varepsilon}} \leq H(u) \leq \frac{C_{\varepsilon}}{u^{\alpha-\varepsilon}}, \\
& \frac{c_{\varepsilon}}{u^{\frac{1}{\alpha}+\varepsilon}} \leq \phi_{u} \leq \frac{C_{\varepsilon}}{u^{\frac{1}{\alpha}-\varepsilon}} .
\end{aligned}
$$

The following Lemma gives useful asymptotics of the truncated moments of the Lévy measure $v$.

Lemma 3.1 1. For $\alpha \in(0,1]$ and any $\varepsilon>0$ there is $C(\varepsilon)>0$ such that

$$
\int_{1<|z| \leq R}|z| v(\mathrm{~d} z) \leq C(\varepsilon) R^{1-\alpha+\varepsilon} .
$$

2. For $\alpha \in(1,2)$ there is $C>0$ such that

$$
\int_{1<|z| \leq R}|z| v(\mathrm{~d} z) \leq C .
$$

3. For $\alpha \in(0,2)$ and any $\varepsilon>0$ there is $C(\varepsilon)>0$ such that

$$
\int_{1<|z| \leq R} z^{2} v(\mathrm{~d} z) \leq C(\varepsilon) R^{2-\alpha+\varepsilon} .
$$

Proof To prove the first inequality we integrate by parts and note that for any $\varepsilon>0$

$$
\begin{aligned}
\int_{1<|z| \leq R}|z| v(\mathrm{~d} z)=-\int_{(1, R]} z \mathrm{~d} H(z)= & -\left.z H(z)\right|_{1} ^{R}+\int_{(1, R]} H(z) \mathrm{d} z \\
& \leq H(1)+C_{\varepsilon} \int_{1}^{R} \frac{\mathrm{d} z}{z^{\alpha-\varepsilon}} .
\end{aligned}
$$

Hence (3.3) follows for any $\varepsilon>0$ and (3.4) is obtained if we choose $\varepsilon \in(0, \alpha-1)$. The estimate (3.5) is obtained analogously to (3.3).

The next Lemma will be used to determine the tail behaviour of the product of any two independent normalized jumps $\left|J_{k}^{T}\right|\left|J_{l}^{T}\right| / R_{T}^{2}, k \neq l$.

Lemma 3.2 Let $U_{R} \geq 1$ and $V_{R} \geq 1$ be two independent random variables with probability distribution function

$$
\mathbf{P}\left(U_{R}>x\right)=\mathbf{P}\left(V_{R}>x\right)=\bar{F}_{R}(x)=\frac{H(x R)}{H(R)}, \quad R \geq 1, \quad x \geq 1 .
$$

Then for each $\varepsilon \in(0, \alpha)$ there is $C(\varepsilon)>0$ such that for all $R \geq 1$ and all $x \geq 1$

$$
\mathbf{P}\left(U_{R} V_{R}>x\right) \leq \frac{C(\varepsilon)}{x^{\alpha-\varepsilon}} .
$$


Proof Recall that Potter's bounds Resnick (2007, Proposition 2.6 (ii)) imply that for each $\varepsilon>0$ there is $C_{0}(\varepsilon)>0$ such that for each $x \geq 1$ and $R \geq 1$

$$
\bar{F}_{R}(x)=\frac{H(x R)}{H(R)} \leq \frac{C_{0}(\varepsilon)}{x^{\alpha-\varepsilon}} .
$$

Moreover,

$$
\bar{F}_{R}(x) \equiv 1, \quad x \in[0,1] .
$$

For $x>1$ we write

$$
\begin{aligned}
\mathbf{P}\left(U_{R} V_{R}>x\right) & =\int_{1}^{\infty} \int_{x / u}^{\infty} \mathrm{d} F_{R}(v) \mathrm{d} F_{R}(u) \\
& =\left(\int_{1}^{x}+\int_{x}^{\infty}\right) \bar{F}_{R}(x / u) \mathrm{d} F_{R}(u)=I_{R}^{(1)}(x)+I_{R}^{(2)}(x) .
\end{aligned}
$$

Then

$$
I_{R}^{(2)}(x)=\int_{x}^{\infty} \bar{F}_{R}(x / u) \mathrm{d} F_{R}(u) \leq \int_{x}^{\infty} \mathrm{d} F_{R}(u) \leq \bar{F}_{R}(x) \leq \frac{C_{0}(\varepsilon)}{x^{\alpha-\varepsilon}} .
$$

Eventually,

$$
\begin{aligned}
I_{R}^{(1)}(x) & \leq \frac{C_{0}(\varepsilon)}{x^{\alpha-\varepsilon}} \int_{1}^{x} u^{\alpha-\varepsilon} \mathrm{d} F_{R}(u)=-\frac{C_{0}(\varepsilon)}{x^{\alpha-\varepsilon}} \int_{1}^{x} u^{\alpha-\varepsilon} \mathrm{d} \bar{F}_{R}(u) \\
& =-\left.\frac{C_{0}(\varepsilon)}{x^{\alpha-\varepsilon}} u^{\alpha-\varepsilon} \bar{F}_{R}(u)\right|_{1} ^{x}+(\alpha-\varepsilon) \frac{C_{0}(\varepsilon)}{x^{\alpha-\varepsilon}} \int_{1}^{x} u^{\alpha-1-\varepsilon} \bar{F}_{R}(u) \mathrm{d} u \\
& \leq \frac{C_{0}(\varepsilon)}{x^{\alpha-\varepsilon}}+(\alpha-\varepsilon) \frac{C_{0}(\varepsilon)^{2}}{x^{\alpha-\varepsilon}} \int_{1}^{x} \frac{u^{\alpha-1-\varepsilon}}{u^{\alpha-\varepsilon}} \mathrm{d} u \\
& \leq \frac{C_{0}(\varepsilon)}{x^{\alpha-\varepsilon}}+(\alpha-\varepsilon) \frac{C_{0}(\varepsilon)^{2}}{x^{\alpha-\varepsilon}} \ln x \\
& \leq \frac{C(\varepsilon)}{x^{\alpha-2 \varepsilon}}
\end{aligned}
$$

for some $C(\varepsilon)>0$.

Remark 3.3 A finer tail asymptotics of products of iid non-negative Pareto type random variables can be found in Rosiński and Woyczyński (1987, Theorem 2.1) and Jessen and Mikosch (2006, Lemma 4.1 (4)). In Lemma 3.2, however, we establish rather rough estimates which are valid for the families of iid random variables $\left\{U_{R}, V_{R}\right\}_{R \geq 1}$.

The following useful Lemma will be used to determine the conditional distribution of the interarrival times of the compound Poisson process $\eta^{T}$.

Lemma 3.4 Let $T>0$ and let $N=\left(N_{t}\right)_{t \in[0, T]}$ be a Poisson process, $\left\{\tau_{k}\right\}_{k \geq 1}$ be its arrival times, $\tau_{0}=0$. Then for each $m \geq 1$, and $1 \leq j<j+k \leq m$

$$
\mathbf{P}\left(\tau_{j+k}-\tau_{j} \leq s \mid N_{T}=m\right)=\mathbf{P}\left(\sigma_{k} \leq \frac{s}{T}\right), \quad s \in[0,1],
$$

where $\sigma_{k}$ is a $\operatorname{Beta}(m, k-1)$-distributed random variable with density

$$
f_{\sigma_{k}}^{(m)}(u)=\frac{m !}{(k-1) !(m-k) !} u^{k-1}(1-u)^{m-k}, \quad u \in[0,1], \quad m \geq 1,1 \leq k \leq m .
$$


Proof It is well known that the conditional distribution of the arrival times $\tau_{1}, \ldots, \tau_{m}$, given that $N_{T}=m$, coincides with the distribution of the order statistics obtained from $m$ samples from the population with uniform distribution on [0,T], see Sato (1999, Proposition 3.4).

Let, for brevity, $T=1$. The joint density of $\left(\tau_{j}, \tau_{j+k}\right), 1 \leq j<j+k \leq m$ is well known, see e.g. Balakrishnan and Nevzorov (2003, Chapter 11.10):

$$
\begin{aligned}
f_{\tau_{j}, \tau_{j+k}}^{(m)}(u, v) & =c_{j, k, m} \cdot u^{j-1}(v-u)^{k-1}(1-v)^{m-j-k} \mathbb{I}(0 \leq u<v \leq 1), \\
c_{j, k, m} & =\frac{m !}{(j-1) !(k-1) !(m-j-k) !},
\end{aligned}
$$

and consequently

$$
f_{\tau_{j+k}-\tau_{j}, \tau_{j}}^{(m)}(u, v)=c_{j, k, m} \cdot v^{j-1} u^{k-1}(1-u-v)^{m-j-k}, \quad u, v, u+v \in[0,1] .
$$

Hence, the probability density of the difference $\tau_{j+k}-\tau_{j}$ is obtained by integration w.r.t. $v \in[0,1]$,

$$
\begin{aligned}
f_{\tau_{j+k}-\tau_{j}}^{(m)}(u)= & c_{k, j, m} \cdot u^{k-1} \int_{0}^{1-u} v^{j-1}(1-u-v)^{m-j-k} \mathrm{~d} v \\
& \stackrel{(1-u) z}{=} c_{j, k, m} \cdot u^{k-1} \cdot(1-u)^{m-k} \int_{0}^{1} z^{j-1}(1-z)^{m-j-k} \mathrm{~d} z .
\end{aligned}
$$

Recalling the definition of the Beta-function, we get

$$
\int_{0}^{1} z^{j-1}(1-z)^{m-j-k} \mathrm{~d} z=\frac{(j-1) !(m-j-k) !}{(m-k) !},
$$

which yields the desired result.

Lemma 3.5 Let $\boldsymbol{A}_{v}$ hold true and $\left\{\phi_{T}\right\}$ be the scaling defined in (2.5). Then for any $\rho \in\left[0, \frac{1}{\alpha}\right)$

$$
\phi_{T}^{2}\left[\eta^{T}\right]_{T} \stackrel{\mathrm{d}}{\rightarrow} \mathcal{S}^{(\alpha / 2)}, \quad T \rightarrow \infty
$$

where $\mathcal{S}^{(\alpha / 2)}$ is a spectrally positive $\alpha / 2$-stable random variable with Laplace transform (2.6).

Proof The process $t \mapsto \phi_{T}^{2}\left[\eta^{T}\right]_{t}$ is a compound Poisson process with Lévy measure $v_{T}$ with the tail

$$
H_{T}(u)=\int_{u}^{\infty} v_{T}(\mathrm{~d} z)=H\left(\frac{\sqrt{u}}{\phi_{T}} \vee R_{T}\right), \quad u>0 .
$$

The Laplace transform of $\phi_{T}^{2}\left[\eta^{T}\right]_{T}$ has the cumulant

$$
K_{T}(\lambda):=\ln \mathbf{E e}^{-\lambda \phi_{T}^{2}\left[\eta^{T}\right]_{T}}=-T \int_{0}^{\infty}\left(\mathrm{e}^{-\lambda u}-1\right) \mathrm{d} H_{T}(u), \quad \lambda \geq 0 .
$$

Integrating by parts yields

$$
K_{T}(\lambda)=-\left.T\left(\mathrm{e}^{-\lambda u}-1\right) H_{T}(u)\right|_{0} ^{\infty}-\lambda T \int_{0}^{\infty} \mathrm{e}^{-\lambda u} H_{T}(u) \mathrm{d} u .
$$


Since the first summands on the r.h.s. of (3.8) vanish, it is left to evaluate the integral term. Taking into account (2.5), namely that $\frac{1}{T}=\tilde{H}\left(\frac{1}{\phi_{T}}\right)$, we write for any $u_{0}>0$

$$
\begin{aligned}
K_{T}(\lambda)=-\lambda T \int_{0}^{\infty} \mathrm{e}^{-\lambda u} H_{T}(u) \mathrm{d} u \\
=-\lambda T \int_{0}^{u_{0}} \mathrm{e}^{-\lambda u} H\left(\frac{\sqrt{u}}{\phi_{T}} \vee R_{T}\right) \mathrm{d} u \\
\quad \quad-\lambda \frac{H\left(1 / \phi_{T}\right)}{\tilde{H}\left(1 / \phi_{T}\right)} \frac{1}{H\left(1 / \phi_{T}\right)} \int_{u_{0}}^{\infty} \mathrm{e}^{-\lambda u} H\left(\frac{\sqrt{u}}{\phi_{T}} \vee R_{T}\right) \mathrm{d} u \\
=-I_{T}^{(1)}(\lambda)-I_{T}^{(2)}(\lambda) .
\end{aligned}
$$

It is evident that $\lim _{T \rightarrow \infty} \frac{H\left(1 / \phi_{T}\right)}{\tilde{H}\left(1 / \phi_{T}\right)}=1$. Moreover for $\rho \in[0,1 / \alpha)$ due to Resnick (2007, Proposition 2.4), the convergence

$$
\lim _{T \rightarrow \infty} \frac{H\left(\frac{\sqrt{u}}{\phi_{T}} \vee R_{T}\right)}{H\left(\frac{1}{\phi_{T}}\right)}=\frac{1}{u^{\alpha / 2}}
$$

holds uniformly on each half-line $\left[u_{0}, \infty\right), u_{0}>0$, and thus for each $u_{0}>0$

$$
\lim _{T \rightarrow \infty} I_{T}^{(2)}(\lambda)=\lambda \int_{u_{0}}^{\infty} \frac{\mathrm{e}^{-\lambda u}}{u^{\alpha / 2}} \mathrm{~d} u .
$$

Further we estimate

$$
I_{T}^{(1)}(\lambda) \leq 2 \lambda T \phi_{T}^{2} \int_{0}^{\sqrt{u_{0}} / \phi_{T}} y H(y) \mathrm{d} y .
$$

Note that $y \mapsto y H(y)$ is integrable at 0 by the definition of the Lévy measure, $0 \leq$ $-\int_{0}^{1} y^{2} \mathrm{~d} H(y)<\infty$, and the integration by parts. Eventually by Karamata's theorem (Resnick 2007, Theorem 2.1 (a))

$$
\begin{aligned}
I_{T}^{(1)} \leq & 2 \lambda \frac{H\left(1 / \phi_{T}\right)}{\tilde{H}\left(1 / \phi_{T}\right)} \cdot \frac{\phi_{T}^{2}}{H\left(1 / \phi_{T}\right)} \cdot \frac{\int_{0}^{\sqrt{u_{0}} / \phi_{T}} y H(y) \mathrm{d} y}{\frac{u_{0}}{\phi_{T}^{2}} H\left(\frac{\sqrt{u_{0}}}{\phi_{T}}\right)} \cdot \frac{u_{0}}{\phi_{T}^{2}} \cdot H\left(\frac{\sqrt{u_{0}}}{\phi_{T}}\right) \\
& \rightarrow \frac{2 \lambda}{2-\alpha} u_{0}^{1-\frac{\alpha}{2}}, \quad T \rightarrow \infty .
\end{aligned}
$$

Hence choosing $u_{0}>0$ sufficiently small and letting $T \rightarrow \infty$ we obtain the convergence of $K_{T}$ to the cumulant of a spectrally positive stable random variable

$$
\lim _{T \rightarrow \infty} K_{T}(\lambda)=-\lambda \int_{0}^{\infty} \frac{\mathrm{e}^{-\lambda u}}{u^{\alpha / 2}} \mathrm{~d} u=-\Gamma\left(1-\frac{\alpha}{2}\right) \lambda^{\alpha / 2} .
$$

Lemma 3.6 For any $\rho \in[0,1 / \alpha)$ and any $\theta>0$

$$
\begin{gathered}
\phi_{T}^{2}\left|X_{T}^{T}\right|^{2} \stackrel{\mathrm{d}}{\rightarrow} 0, \quad T \rightarrow \infty, \\
\phi_{T}^{2} \int_{0}^{T}\left|X_{s}^{T}\right|^{2} \mathrm{~d} s \stackrel{\mathrm{d}}{\rightarrow} 0, \quad T \rightarrow \infty .
\end{gathered}
$$


Proof

$$
\begin{aligned}
\left|X_{s}^{T}\right|^{2} & \leq 3\left|X_{0}\right|^{2} \mathrm{e}^{-2 \theta s}+3 \mathrm{e}^{-2 \theta s}\left|\int_{0}^{s} \mathrm{e}^{\theta r} \mathrm{~d} \xi_{r}^{T}\right|^{2}+3 b_{T}^{2} \mathrm{e}^{-2 \theta s}\left|\int_{0}^{s} \mathrm{e}^{\theta r} \mathrm{~d} r\right|^{2} \\
& =a_{1}(s)+a_{2}(s)+a_{3}(s) .
\end{aligned}
$$

By the Itô isometry and Lemma 3.1, for any $\varepsilon>0$ we estimate for each $s \geq 0$

$$
\begin{aligned}
\phi_{T}^{2} \mathbf{E} a_{2}(s) & =\phi_{T}^{2} \cdot \frac{3}{2 \theta}\left(\sigma^{2}+\int_{|z| \leq R_{T}} z^{2} v(\mathrm{~d} z)\right) \cdot \mathrm{e}^{-2 \theta s}\left(\mathrm{e}^{2 \theta s}-1\right) \\
& \leq C_{1} \cdot T^{-\frac{2}{\alpha}+\varepsilon+\rho(2-\alpha+\varepsilon)} .
\end{aligned}
$$

Analogously, Lemma 3.1 yields

$$
b_{T}^{2} \leq \begin{cases}C_{2} T^{2 \rho(1-\alpha+\varepsilon)}, & \alpha \in(0,1] \\ C_{2}, & \alpha \in(1,2) .\end{cases}
$$

and hence for each $s \geq 0$

$$
\phi_{T}^{2} \cdot a_{3}(s) \leq C_{3} \max \left\{1, T^{2 \rho(1-\alpha+\varepsilon)}\right\} \cdot T^{-\frac{2}{\alpha}+\varepsilon} \rightarrow 0 .
$$

Finally, for $s \geq 0$

$$
\phi_{T}^{2} a_{1}(s) \leq C_{4}\left|X_{0}\right|^{2} \cdot T^{-\frac{2}{\alpha}+\varepsilon} \rightarrow 0 \quad \text { a.s. as } T \rightarrow+\infty .
$$

For any $\rho \in[0,1 / \alpha)$ we can choose $\varepsilon>0$ sufficiently small such that the bounds in (3.10) and (3.11) and (3.12) converge to 0 as $T \rightarrow \infty$ which gives (3.9). Integrating these inequalities w.r.t. $s \in[0, T]$ results in an additional factor $T$ on the r.h.s. of these estimates, and convergence to 0 still holds true for $\varepsilon>0$ sufficiently small.

Lemma 3.7 For any $\rho>\frac{1}{2 \alpha}$ and any $\theta>0$

$$
\phi_{T}^{2} \int_{0}^{T} X_{s-}^{\eta^{T}} \mathrm{~d} \eta_{s}^{T} \stackrel{\mathrm{d}}{\rightarrow} 0, \quad T \rightarrow \infty .
$$

Proof The Ornstein-Uhlenbeck process $X^{\eta^{T}}$ as well as its integral w.r.t. $\eta^{T}$ can be written explicitly in the form of sums:

$$
\begin{aligned}
X_{t}^{\eta^{T}} & =\sum_{j=1}^{\infty} J_{j}^{T} \mathrm{e}^{-\theta\left(t-\tau_{j}^{T}\right)} \mathbb{I}_{\left[\tau_{j}^{T}, \infty\right)}(t), \\
X_{\tau_{k}^{T}-}^{\eta^{T}} & =\sum_{j=1}^{k-1} J_{j}^{T} \mathrm{e}^{-\theta\left(\tau_{k}^{T}-\tau_{j}^{T}\right)}, \quad k \geq 1, \\
\int_{0}^{T} X_{s-}^{\eta^{T}} \mathrm{~d} \eta_{s}^{T} & =\sum_{k=1}^{N_{T}^{T}} X_{\tau_{k}^{T}-}^{\eta^{T}} J_{k}^{T}=\sum_{k=1}^{N_{T}^{T}} J_{k}^{T} \sum_{j=1}^{k-1} J_{j}^{T} \mathrm{e}^{-\theta\left(\tau_{k}^{T}-\tau_{j}^{T}\right)} .
\end{aligned}
$$

As always, we agree that $\sum_{j=k}^{m}=0$ for $m<k$.

Note that for $N_{T}^{T}=0$ and $N_{T}^{T}=1, \int_{0}^{T} X_{s-}^{\eta^{T}} \mathrm{~d} \eta_{s}^{T}=0$. For $m \geq 2$, on the event $N_{T}^{T}=m$ we get the estimate

$$
\left|\int_{0}^{T} X_{s-}^{\eta^{T}} \mathrm{~d} \eta_{s}^{T}\right| \leq \sum_{k=2}^{m}\left|J_{k}^{T}\right| \sum_{j=1}^{k-1}\left|J_{j}^{T}\right| \mathrm{e}^{-\theta\left(\tau_{k}^{T}-\tau_{j}^{T}\right)}=\sum_{j=1}^{m-1} \sum_{k=1}^{m-j}\left|J_{j+k}^{T}\right|\left|J_{j}^{T}\right| \mathrm{e}^{-\theta\left(\tau_{j+k}^{T}-\tau_{j}^{T}\right)} .
$$


We also take into account that for all $m \geq 2$ and $1 \leq j<j+k \leq m$

$$
\operatorname{Law}\left(\left|J_{j+k}^{T}\right|\left|J_{j}^{T}\right| \mathrm{e}^{-\theta\left(\tau_{j+k}^{T}-\tau_{j}^{T}\right)} \mid N_{T}^{T}=m\right) \stackrel{\mathrm{d}}{=} \operatorname{Law}\left(R_{T}^{2} \cdot U_{T} \cdot V_{T} \cdot \mathrm{e}^{-\theta T \sigma_{k}}\right),
$$

where $U_{T}, V_{T}$ are iid random variables with probability law

$$
\mathbf{P}\left(U_{T} \geq x\right)=\frac{H\left(x R_{T}\right)}{H\left(R_{T}\right)}, \quad x \geq 1,
$$

and $\sigma_{k}, k=1, \ldots, m-1$, is a $\operatorname{Beta}(k, m-1+k)$-distributed random variable independent of $U_{T}$ and $V_{T}$ with probability density (3.7). For each $m \geq 0$ denote by $\mathbf{P}_{T}^{(m)}$ the conditional law $\mathbf{P}\left(\cdot \mid N_{T}^{T}=m\right)$.

For some $\varepsilon \in\left(0, \frac{2-\alpha}{\alpha}\right)$ which will be chosen sufficiently small later, and for each $m \geq 2$ define the family of positive weights

$$
w_{k, m}=\left(C(\alpha, \varepsilon) \cdot(m-1) \cdot k^{\frac{2}{\alpha}-\varepsilon}\right)^{-1}, \quad k=1, \ldots, m-1,
$$

where

$$
C(\alpha, \varepsilon)=\sum_{k=1}^{\infty} k^{-\frac{2}{\alpha}+\varepsilon}<\infty
$$

is the normalizing constant. With this construction for each $m \geq 2$

$$
\sum_{k=1}^{m-1} \sum_{j=1}^{m-k} w_{k, m}=\sum_{k=1}^{m-1}(m-k) w_{k, m}=\frac{1}{C(\alpha, \varepsilon)} \sum_{k=1}^{m-1} \frac{m-k}{m-1} \cdot k^{-\frac{2}{\alpha}+\varepsilon} \leq 1 .
$$

Let $\gamma>0$. In order to show that the sum (3.13) multiplied by $\phi_{T}^{2}$ converges to zero, we take into account (3.14) and write

$$
\begin{aligned}
\mathbf{P}_{T}^{(m)} & \left(\phi_{T}^{2} \sum_{j=1}^{m-1} \sum_{k=1}^{m-j}\left|J_{j+k}^{T}\right|\left|J_{j}^{T}\right| \mathrm{e}^{-\theta\left(\tau_{j+k}-\tau_{j}\right)}>\gamma\right) \\
\leq & \mathbf{P}_{T}^{(m)}\left(\phi_{T}^{2} \sum_{k=1}^{m-1} \sum_{j=1}^{m-k}\left|J_{j+k}^{T}\right|\left|J_{j}^{T}\right| \mathrm{e}^{-\theta\left(\tau_{j+k}-\tau_{j}\right)}>\gamma \sum_{k=1}^{m-1} \sum_{j=1}^{m-k} w_{k, m}\right) \\
\leq & \sum_{k=1}^{m-1} \sum_{j=1}^{m-k} \mathbf{P}\left(\phi_{T}^{2} R_{T}^{2} U_{T} V_{T} \mathrm{e}^{-\theta T \sigma_{k}}>\gamma w_{k, m}\right) \\
& =\sum_{k=1}^{m-1}(m-k) \mathbf{P}\left(\phi_{T}^{2} R_{T}^{2} \cdot U_{T} V_{T} \mathrm{e}^{-\theta T \sigma_{k}}>\gamma w_{k, m}\right) .
\end{aligned}
$$


Applying Lemma 3.2 and the independence of $U_{T} V_{T}$ and $\sigma_{k}$ we obtain for some $\varepsilon>0$

$$
\begin{aligned}
p_{k, m}(T) & =\mathbf{P}\left(\phi_{T}^{2} R_{T}^{2} \cdot U_{T} V_{T} \mathrm{e}^{-\theta T \sigma_{k}}>\gamma w_{k, m}\right) \\
& =\frac{m !}{(k-1) !(m-k) !} \int_{0}^{1} \mathbf{P}\left(U_{T} V_{T}>\gamma \frac{w_{k, m}}{\phi_{T}^{2} R_{T}^{2}} \cdot \mathrm{e}^{\theta T u}\right)(1-u)^{m-k} u^{k-1} \mathrm{~d} u \\
& \leq C(\varepsilon) \frac{m !}{(k-1) !(m-k) !}\left(\gamma \frac{w_{k, m}}{\phi_{T}^{2} R_{T}^{2}}\right)^{-\alpha+\varepsilon} \int_{0}^{1} \mathrm{e}^{-\theta T u(\alpha-\varepsilon)}(1-u)^{m-k} u^{k-1} \mathrm{~d} u \\
& \leq C(\varepsilon) \frac{m !}{(k-1) !(m-k) !}\left(\gamma \frac{w_{k, m}}{\phi_{T}^{2} R_{T}^{2}}\right)^{-\alpha+\varepsilon} \int_{0}^{\infty} \mathrm{e}^{-\theta T u(\alpha-\varepsilon)} u^{k-1} \mathrm{~d} u \\
& \leq C(\varepsilon, \alpha, \gamma) \frac{m !}{(k-1) !(m-k) !}\left(k^{\frac{2}{\alpha}-\varepsilon}(m-1) T^{-\frac{2}{\alpha}+2 \rho+\varepsilon}\right)^{\alpha-\varepsilon} \frac{(k-1) !}{(\theta T(\alpha-\varepsilon))^{k}} \\
& \leq C(\varepsilon, \alpha, \gamma) \cdot T^{\left(-\frac{2}{\alpha}+2 \rho+\varepsilon\right)(\alpha-\varepsilon)} \cdot \frac{m ! m^{2} k^{2}}{(m-k) !} \cdot \frac{1}{(\theta T(\alpha-\varepsilon))^{k}},
\end{aligned}
$$

where we have used the well known relation $\int_{0}^{\infty} a u^{n} \mathrm{e}^{-a u} \mathrm{~d} u=n ! / a^{n}, a>0, n \geq 0$, as well as the elementary estimates $(m-1)^{\alpha-\varepsilon} \leq m^{2}$ and $k^{\left(\frac{2}{\alpha}-\varepsilon\right)(\alpha-\varepsilon)} \leq k^{2}$ which are valid for $\varepsilon>0$ and $\alpha \in(0,2)$.

Hence

$$
\begin{aligned}
p(T):= & \sum_{m=2}^{\infty}\left[\mathbf{P}\left(N_{T}^{T}=m\right) \sum_{k=1}^{m-1}(m-k) p_{k, m}(T)\right] \\
= & C(\varepsilon, \alpha, \gamma) \cdot T^{\left(-\frac{2}{\alpha}+2 \rho+\varepsilon\right)(\alpha-\varepsilon)} \\
& \times \sum_{m=2}^{\infty}\left[\mathrm{e}^{-T \cdot H\left(R_{T}\right)} \frac{\left(T \cdot H\left(R_{T}\right)\right)^{m}}{m !} \sum_{k=1}^{m-1}(m-k) \frac{m ! m^{2} k^{2}}{(m-k) !} \cdot \frac{1}{(\theta T(\alpha-\varepsilon))^{k}}\right] \\
= & C(\varepsilon, \alpha, \gamma) \cdot \mathrm{e}^{-T \cdot H\left(R_{T}\right)} \cdot T^{\left(-\frac{2}{\alpha}+2 \rho+\varepsilon\right)(\alpha-\varepsilon)} \\
& \quad \times \sum_{k=1}^{\infty} \frac{k^{2}}{(\theta T(\alpha-\varepsilon))^{k}} \sum_{m=k+1}^{\infty} m^{2} \frac{\left(T \cdot H\left(R_{T}\right)\right)^{m}}{(m-k-1) !} .
\end{aligned}
$$

To evaluate the inner sum we use the formula $\sum_{j=0}^{\infty}(j+k)^{2} a^{j} / j !=\mathrm{e}^{a}\left(a^{2}+2 a k+a+k^{2}\right)$ to obtain

$$
\begin{aligned}
& \sum_{m=k+1}^{\infty} m^{2} \frac{\left(T \cdot H\left(R_{T}\right)\right)^{m}}{(m-k-1) !} \\
& \quad=\left(T \cdot H\left(R_{T}\right)\right)^{k+1} \sum_{j=0}^{\infty}(j+k+1)^{2} \frac{\left(T \cdot H\left(R_{T}\right)\right)^{j}}{j !} \\
& \quad \leq 3\left(\left(T \cdot H\left(R_{T}\right)\right)^{k+3}+(k+1)^{2}\left(T \cdot H\left(R_{T}\right)\right)^{k+1}\right) \mathrm{e}^{T \cdot H\left(R_{T}\right)} .
\end{aligned}
$$

Combining (3.15) and (3.16), it is left to estimate two summands. For the first one, we use the formula $\sum_{k=1}^{\infty} k^{2} q^{k}=q(q+1) /(1-q)^{3},|q|<1$, to get

$$
S_{1}=\sum_{k=1}^{\infty} \frac{k^{2}}{(\theta T(\alpha-\varepsilon))^{k}}\left(T \cdot H\left(R_{T}\right)\right)^{k+3} \leq C_{1} \cdot T^{3} \cdot H\left(R_{T}\right)^{4} .
$$


For the second summand, we use the formula $\sum_{k=1}^{\infty} k^{2}(k+1)^{2} q^{k}=4 q\left(q^{2}+4 q+1\right) /(1-q)^{5}$, $|q|<1$, to get

$$
S_{2}=\sum_{k=1}^{\infty} \frac{k^{2}(k+1)^{2}}{(\theta T(\alpha-\varepsilon))^{k}}\left(T \cdot H\left(R_{T}\right)\right)^{k+1} \leq C_{2} \cdot T \cdot H\left(R_{T}\right)^{2} .
$$

Combining (3.15) with the bounds for $S_{1}$ and $S_{2}$ we obtain

$$
p(T) \leq C \cdot T^{\left(-\frac{2}{\alpha}+2 \rho+\varepsilon\right)(\alpha-\varepsilon)} \cdot\left(T^{3-4 \rho(\alpha-\varepsilon)}+T^{1-2 \alpha \rho+\varepsilon}\right) .
$$

Since $\rho>\frac{1}{2 \alpha}$, one can choose $\varepsilon>0$ sufficiently small to obtain the limit $p(T) \rightarrow 0$, $T \rightarrow \infty$.

\section{Proofs of the main results}

Proof of Theorem 2.6 Let $\rho \in\left(\frac{1}{2 \alpha}, \frac{1}{\alpha}\right)$ be fixed. With the help of the decomposition (3.1) we may write

$$
\int_{0}^{T} X_{s}^{2} \mathrm{~d} s=\int_{0}^{T}\left(X_{s}^{\eta^{T}}\right)^{2} \mathrm{~d} s+\int_{0}^{T}\left(X_{s}^{T}\right)^{2} \mathrm{~d} s+2 \int_{0}^{T} X_{s}^{T} \cdot X_{s}^{\eta^{T}} \mathrm{~d} s .
$$

Then by Lemma 3.6, $\phi_{T}^{2} \int_{0}^{T}\left(X_{s}^{T}\right)^{2} \mathrm{~d} s \stackrel{\mathrm{d}}{\rightarrow} 0$. Recall that $X^{\eta^{T}}$ satisfies the SDE

$$
\mathrm{d} X_{t}^{\eta^{T}}=-\theta X_{t}^{\eta^{T}} \mathrm{~d} t+\mathrm{d} \eta_{t}^{T}, \quad X_{0}^{\eta^{T}}=0 .
$$

The Itô formula applied to the process $X^{\eta^{T}}$ yields

$$
\left(X_{T}^{\eta^{T}}\right)^{2}=-2 \theta \int_{0}^{T}\left(X_{s}^{\eta^{T}}\right)^{2} \mathrm{~d} s+2 \int_{0}^{T} X_{s-}^{\eta^{T}} \mathrm{~d} \eta_{s}^{T}+\left[\eta^{T}\right]_{T} .
$$

The decomposition (3.1) implies that $\left(X_{T}^{\eta^{T}}\right)^{2} \leq 2 X_{T}^{2}+2\left(X_{T}^{T}\right)^{2}$. Since for $\theta>0$ the process $X$ has an invariant distribution (see, e.g. Sato 1999, Theorem 17.5 and Remark 2.3), we get that $\phi_{T}^{2} X_{T}^{2} \rightarrow 0$ in law. On the other hand, $\phi_{T}^{2}\left(X_{T}^{T}\right)^{2} \rightarrow 0$ in law by Lemma 3.6. Therefore, Lemmas 3.5, 3.7 and (4.2) yield

$$
\phi_{T}^{2} \int_{0}^{T}\left(X_{s}^{\eta^{T}}\right)^{2} \mathrm{~d} s \stackrel{\mathrm{d}}{\rightarrow} \frac{\mathcal{S}^{(\alpha / 2)}}{2 \theta}, \quad T \rightarrow \infty
$$

Eventually, the last integral in (4.1) multiplied by $\phi_{T}^{2}$ converges to 0 by the Cauchy-Schwarz inequality.

Proof of Corollary 2.7 The decomposition

$$
X_{t}=X_{t}^{T}+X_{t}^{\eta^{T}}=X_{0} \mathrm{e}^{-\theta t}+b_{T} \frac{1-\mathrm{e}^{-\theta s}}{2 \theta}+\int_{0}^{t} \mathrm{e}^{-\theta(t-s)} \mathrm{d} \xi_{s}^{T}+X_{t}^{\eta^{T}}
$$

allows us to write

$$
\int_{0}^{T} X_{s} \mathrm{~d} W_{s}=\int_{0}^{T} X_{s}^{T} \mathrm{~d} W_{s}+\int_{0}^{T} X_{s}^{\eta^{T}} \mathrm{~d} W_{s}
$$


as well as (4.1). It is easy to check that $\phi_{T} \int_{0}^{T} X_{s}^{T} \mathrm{~d} W_{S} \stackrel{\mathrm{d}}{\rightarrow} 0$. Indeed, due to the independence of $X_{0}$ and $W$

$$
\phi_{T} \int_{0}^{T} X_{0} \mathrm{e}^{-\theta s} \mathrm{~d} W_{s}=\phi_{T} \cdot X_{0} \cdot \int_{0}^{T} \mathrm{e}^{-\theta s} \mathrm{~d} W_{s} \rightarrow 0 \text { a.s. }
$$

and obviously by Lemma 3.7

$$
\phi_{T} b_{T} \int_{0}^{T}\left(1-\mathrm{e}^{-\theta s}\right) \mathrm{d} W_{s} \stackrel{\mathrm{d}}{\rightarrow} 0 .
$$

Finally by the estimate (3.10) of Lemma 3.6

$$
\begin{aligned}
\mathbf{E}\left[\phi_{T} \int_{0}^{T}\left(X_{s}^{T}-X_{0} \mathrm{e}^{-\theta s}-b_{T} \frac{1-\mathrm{e}^{-\theta s}}{2 \theta}\right) \mathrm{d} W_{s}\right]^{2} \\
=\phi_{T}^{2} \mathbf{E}\left[\int_{0}^{T} \int_{0}^{s} \mathrm{e}^{-\theta(s-r)} \mathrm{d} \xi_{r}^{T} \mathrm{~d} W_{s}\right]^{2} \\
=\phi_{T}^{2} \int_{0}^{T} \mathbf{E}\left[\int_{0}^{s} \mathrm{e}^{-\theta(s-r)} \mathrm{d} \xi_{r}^{T}\right]^{2} \mathrm{~d} s \\
=\phi_{T}^{2} \int_{0}^{T} \int_{0}^{s} \mathrm{e}^{-2 \theta(s-r)} \mathrm{d} r \mathrm{~d} s \cdot\left(\sigma^{2}+\int_{|z| \leq R_{T}} z^{2} v(\mathrm{~d} z)\right) \rightarrow 0, \quad T \rightarrow \infty .
\end{aligned}
$$

Taking into account the argument in the proof of Theorem 2.6, we conclude that it is sufficient to consider the limiting behaviour of the pair $\left(\phi_{T} \int_{0}^{T} X_{s}^{\eta^{T}} \mathrm{~d} W_{s}, \phi_{T}^{2} \int_{0}^{T}\left(X_{s}^{\eta^{T}}\right)^{2} \mathrm{~d} s\right)$.

The processes $\eta^{T}$ and $W$ are independent and

$$
M_{t}^{T}=\int_{0}^{t} X_{s}^{\eta^{T}} \mathrm{~d} W_{s}, \quad t \geq 0,
$$

is a continuous local martingale with the angle bracket

$$
\left\langle M^{T}\right\rangle_{t}=\int_{0}^{t}\left(X_{s}^{\eta^{T}}\right)^{2} \mathrm{~d} s,
$$

which is independent of $W$. Then for $u, v \in \mathbb{R}$ we get

$$
\begin{aligned}
\mathbf{E} \exp \left(i u \phi_{T} M_{T}^{T}+i v \phi_{T}^{2}\left\langle M^{T}\right\rangle_{T}\right) & =\mathbf{E}\left[\mathbf{E}\left[\exp \left(i u \phi_{T} M_{T}^{T}+i v \phi_{T}^{2}\left\langle M^{T}\right\rangle_{T}\right) \mid \mathscr{F}_{T}^{\eta^{T}}\right]\right. \\
& =\mathbf{E}\left[\exp \left(i v \phi_{T}^{2}\left\langle M^{T}\right\rangle_{T}\right) \mathbf{E}\left[\exp \left(i u \phi_{T} M_{T}^{T}\right) \mid \mathscr{F}_{T}^{\eta^{T}}\right]\right] \\
& =\mathbf{E}\left[\exp \left(i v \phi_{T}^{2}\left\langle M^{T}\right\rangle_{T}\right) \exp \left(-\frac{u^{2}}{2} \phi_{T}^{2}\left\langle M^{T}\right\rangle_{T}\right)\right] \\
& =\mathbf{E} \exp \left(\left(i v-\frac{u^{2}}{2}\right) \phi_{T}^{2}\left\langle M^{T}\right\rangle_{T}\right) \\
& \rightarrow \mathbf{E} \exp \left(\left(i v-\frac{u^{2}}{2}\right) \frac{\mathcal{S}^{(\alpha / 2)}}{2 \theta_{0}}\right) \\
& =\mathbf{E} \exp \left(i u \mathcal{N} \sqrt{\frac{\mathcal{S}^{(\alpha / 2)}}{2 \theta_{0}}}+i v \frac{\mathcal{S}^{(\alpha / 2)}}{2 \theta_{0}}\right), \quad T \rightarrow \infty .
\end{aligned}
$$


Proof of Theorem 2.5 The statement of the theorem follows immediately from Proposition 2.1 and Corollary 2.7. Indeed, for each $\theta_{0}>0$ and $u \in \mathbb{R}$ we use the formula (2.3) for the likelihood ratio as well as semimartingale decompositions (2.1) and (2.2) to conclude that

$$
\begin{aligned}
\ln L_{T}\left(\theta_{0}, \theta_{0}+\phi_{T} u\right)= & -\frac{\phi_{T} u}{\sigma^{2}} \int_{0}^{T} X_{s} \mathrm{~d}\left(\sigma W_{s}\right)-\frac{\left(\phi_{T} u\right)^{2}}{2 \sigma^{2}} \int_{0}^{T} X_{s}^{2} \mathrm{~d} s \\
& =-\frac{u}{\sigma} \cdot \phi_{T} \int_{0}^{T} X_{s} \mathrm{~d} W_{s}-\frac{u^{2}}{2 \sigma^{2}} \cdot \phi_{T}^{2} \int_{0}^{T} X_{s}^{2} \mathrm{~d} s \\
& \stackrel{\mathrm{d}}{\rightarrow}-\frac{u}{\sigma} \cdot \mathcal{N} \sqrt{\frac{\mathcal{S}^{(\alpha / 2)}}{2 \theta_{0}}}-\frac{u^{2}}{2 \sigma^{2}} \cdot \frac{\mathcal{S}^{(\alpha / 2)}}{2 \theta_{0}}, \quad T \rightarrow \infty .
\end{aligned}
$$

Proof of Corollary 2.8 . The relation (2.8) follows from Proposition 2.1. Due to the linearquadratic form of the likelihood ratio, the maximum likelihood estimator coincides with the so-called central sequence. This implies the asymptotic efficiency in the aforementioned sense. The limit (2.9) follows from Corollary 2.7.

Proof of Remark 2.9 For $x>0$,

$$
\mathbf{P}\left(\frac{|\mathcal{N}|}{\sqrt{\mathcal{S}^{(\alpha / 2)}}}>x\right) \leq \mathbf{P}\left(\mathcal{S}^{(\alpha / 2)} \leq x^{\alpha-2}\right)+\mathbf{P}\left(|\mathcal{N}|>x^{\alpha / 2}\right)=p_{1}(x)+p_{2}(x) .
$$

By the well known property of the Gaussian distribution

$$
p_{2}(x) \leq \sqrt{\frac{2}{\pi}} \frac{\mathrm{e}^{-x^{\alpha} / 2}}{x^{\alpha / 2}} .
$$

To estimate $p_{1}(x)$, we apply the exponential Chebyshev inequality to get

$$
\begin{aligned}
p_{1}(x)=\mathbf{P}\left(\mathcal{S}^{(\alpha / 2)} \leq x^{\alpha-2}\right) & \leq \inf _{\lambda>0} \mathrm{e}^{\lambda x^{\alpha-2}} \mathbf{E e}^{-\lambda S^{(\alpha / 2)}} \\
& =\inf _{\lambda>0} \mathrm{e}^{\lambda x^{\alpha-2}-\Gamma\left(1-\frac{\alpha}{2}\right) \lambda^{\alpha / 2}} \leq \exp \left(-C(\alpha) x^{\alpha}\right)
\end{aligned}
$$

for some $C(\alpha)>0$. Hence the estimate (2.10) follows.

Acknowledgements Open Access funding provided by Projekt DEAL. The authors thank the DAAD exchange programme Eastern Partnership for financial support. A.G. thanks Friedrich Schiller University Jena for hospitality. The authors are grateful to the anonymous referees for their valuable comments and careful reading of the manuscript.

Open Access This article is licensed under a Creative Commons Attribution 4.0 International License, which permits use, sharing, adaptation, distribution and reproduction in any medium or format, as long as you give appropriate credit to the original author(s) and the source, provide a link to the Creative Commons licence, and indicate if changes were made. The images or other third party material in this article are included in the article's Creative Commons licence, unless indicated otherwise in a credit line to the material. If material is not included in the article's Creative Commons licence and your intended use is not permitted by statutory regulation or exceeds the permitted use, you will need to obtain permission directly from the copyright holder. To view a copy of this licence, visit http://creativecommons.org/licenses/by/4.0/. 


\section{References}

Applebaum D (2009) Lévy processes and stochastic calculus, vol 116, 2nd edn. Cambridge studies in advanced mathematics. Cambridge University Press, Cambridge

Balakrishnan N, Nevzorov VB (2003) A primer on statistical distributions. Wiley, Hoboken

Bingham NH, Goldie CM, Teugels JL (1987) Regular variation, vol 27. Encyclopedia of mathematics and its applications. Cambridge University Press, Cambridge

Clément E, Gloter A (2015) Local asymptotic mixed normality property for discretely observed stochastic differential equations driven by stable Lévy processes. Stoch Process Appl 125(6):2316-2352

Clément E, Gloter A (2019) Joint estimation for SDE driven by locally stable Lévy processes. Preprint, 2019. HAL Id: hal-02125428

Clément E, Gloter A, Nguyen H (2019) LAMN property for the drift and volatility parameters of a SDE driven by a stable Lévy process. ESAIM: Probab Stat 23:136-175

Gloter A, Loukianova D, Mai H (2018) Jump filtering and efficient drift estimation for Lévy-driven SDEs. Ann Stat 46(4):1445-1480

Höpfner R (2014) Asymptotic statistics: with a view to stochastic processes. Walter de Gruyter, Berlin

Hu Y, Long H (2007) Parameter estimation for Ornstein-Uhlenbeck processes driven by $\alpha$-stable Lévy motions. Commun Stoch Anal 1(2): 1

Hu Y, Long H (2009a) Least squares estimator for Ornstein-Uhlenbeck processes driven by $\alpha$-stable motions. Stoch Process Appl 119(8):2465-2480

$\mathrm{Hu}$ Y, Long H (2009b) On the singularity of least squares estimator for mean-reverting $\alpha$-stable motions. Acta Mathematica Scientia 29(3):599-608

Ivanenko D, Kulik A (2014) LAN property for discretely observed solutions to Lévy driven SDE's. Modern Stoch Theory Appl 1(1):33-47

Jacod J, Shiryaev AN (2003) Limit theorems for stochastic processes, vol 288, 2nd edn. Grundlehren der Mathematischen Wissenschaften. Springer, Berlin

Jessen AH, Mikosch T (2006) Regularly varying functions. Publications de L'Institut Mathematique. Nouvelle série 80(94):171-192

Kawai R (2013) Local asymptotic normality property for Ornstein-Uhlenbeck processes with jumps under discrete sampling. J Theor Probab 26(4):932-967

Kohatsu-Higa A, Nualart E, Tran NK (2017) LAN property for an ergodic diffusion with jumps. Stat: J Theor Appl Stat 51(2):419-454

Küchler U, Sørensen M (1997) Exponential families of stochastic processes. Springer series in statistics. Springer, New York

Le Cam L, Yang GL (2000) Asymptotics in statistics: some basic concepts, 2nd edn. Springer series in statistics. Springer, New York

Long H (2009) Least squares estimator for discretely observed Ornstein-Uhlenbeck processes with small Lévy noises. Stat Probab Lett 79(19):2076-2085

Mai H (2012) Drift estimation for jump diffusions. Ph.D. thesis, Humboldt-Universität zu Berlin. https://doi. org/10.18452/16590

Mai H (2014) Efficient maximum likelihood estimation for Lévy-driven Ornstein-Uhlenbeck processes. Bernoulli 20(2):919-957

Masuda H (2013) Convergence of Gaussian quasi-likelihood random fields for ergodic Lévy driven SDE observed at high frequency. Ann Stat 41(3):1593-1641

Masuda H (2015) Parametric estimation of Lévy processes, vol 2128. Lévy Matters IV. Lecture Notes in Mathematics. Springer, Berlin, pp 179-286

Masuda H (2019) Non-Gaussian quasi-likelihood estimation of SDE driven by locally stable Lévy process. Stoch Process Appl 129(3):1013-1059

Nguyen TTH (2018) Estimation of the jump processes. Ph.D. thesis, Université Paris-Est. HAL Id: tel02127797

Resnick SI (2007) Heavy-tail phenomena: probabilistic and statistical modeling. Springer series in operations research and financial engineering. Springer, New York

Rosiński J, Woyczyński WA (1987) Multilinear forms in Pareto-like random variables and product random measures. Colloquium Mathematicum 1:303-313

Sato K (1999) Lévy processes and infinitely divisible distributions, vol 68. Cambridge studies in advanced mathematics. Cambridge University Press, Cambridge

Sørensen M (1991) Likelihood methods for diffusions with jumps. In: Prabhu NU, Basawa IV (eds) Statistical inference in stochastic processes. Marcel Dekker Inc, New York, pp 67-105

Tran NK (2017) LAN property for an ergodic Ornstein-Uhlenbeck process with Poisson jumps. Commun Stat Theory Methods 46(16):7942-7968 
Uehara Y (2019) Statistical inference for misspecified ergodic Lévy driven stochastic differential equation models. Stoch Process Appl 129(10):4051-4081

Zhang S, Zhang X (2013) A least squares estimator for discretely observed Ornstein-Uhlenbeck processes driven by symmetric $\alpha$-stable motions. Ann Inst Stat Math 65(1):89-103

Publisher's Note Springer Nature remains neutral with regard to jurisdictional claims in published maps and institutional affiliations. 